\title{
Isovaleric Acidemia: A Novel Mutation with Mild Phenotype
}

\author{
K. Matalon ${ }^{1}$, R. Lombardo ${ }^{2}$, K. Fuller ${ }^{3}$ and R. Matalon ${ }^{4, *}$ \\ ${ }^{1}$ Department of Health and Human Performance Houston, University of Houston, TX, USA \\ ${ }^{2}$ School of Medicine, University of Texas Medical Branch, Galveston, TX, USA \\ ${ }^{3}$ Department of Pediatrics, Baylor Scott and White Memorial Hospital, Temple, TX, USA \\ ${ }^{4}$ Department of Pediatrics, University of Texas Medical Branch, Galveston, TX, USA
}

\begin{abstract}
Isovaleric Acidemia (IVA) is considered a severe metabolic disorder with significant morbidity and mortality. It is caused by deficiency of the enzyme Isovaleryl-CoA dehydrogenase (IVD). Early treatment with carnitine and glycine reduces the episodes of the severe metabolic crises. With the advent of neonatal screening, IVD deficiency has shown a spectrum of severity. We describe a novel homozygous mutation, R332L, in the IVD enzyme with a benign phenotype.
\end{abstract}

Keywords: IVA, IVD, Isovalerylcarnitine, Isovalerylglycine, newborn screen.

\section{INTRODUCTION}

Isovaleric academia (IVA) is an autosomal recessive disorder of leucine metabolism caused by deficiency of Isovaleryl - CoA Dehydrogenase (IVD) [1]. The disease was the first organic acid disorder to be recognized due to the offensive odor during metabolic crisis. Prior to the advent of universal newborn screening, IVA was associated with a high rate of morbidity and mortality. In the United States, the incidence of isovaleric acidemia is approximately $1: 250,000$ [2].

Early classification of IVA identified two phenotypes, the most severe of which is an acute neonatal form with symptom onset within the first two weeks of life [3]. Children diagnosed outside of the neonatal period typically present with a general failure to thrive and developmental delay [4]. Both subsets of patients are prone to develop metabolic crises marked by vomiting, lethargy, and coma [2]. These patients require treatment with carnitine, as well as glycine, which conjugates the isovaleric acid; the conjugate is then excreted by the urinary tract. However, the application of global tandem mass spectroscopy (MS/MS) has allowed for the identification of asymptomatic patients with mild biochemical phenotypes leading to the conclusion that IVA has a spectrum of severity [3].

Since the implementation of expanded neonatal screening, the incidence of isovaleric academia has risen substantially [5]. Recognition of individuals with mild IVD deficiency has expanded [3] and increasingly,

*Address correspondence to this author at the Department of Pediatrics, University of Texas Medical Branch, 301 University Blvd, Galveston, TX 775550359, USA; Tel: 409-772-3466; Fax: 409-772-9595;

E-mail: rmatalon@utmb.edu physicians are being called upon to manage asymptomatic babies without clear guidelines for treatment [6].

The discovery of mild phenotypes of usually severe disorders has presented a major therapeutic challenge [7]. Other metabolic diseases such as galactosemia, biotinidase, medium chain acyl-CoA dehydrogenase deficiency (MCAD), phenylketonuria, tyrosinemia, and others, often can be classified by phenotype, genotype, and biochemical manifestations. Such diseases need to be cataloged so physicians can choose the right treatment and avoid undue anxiety on the part of the parents.

Previously, a mild mutation, $932 \mathrm{C} \rightarrow \mathrm{T}(\mathrm{A} 282 \mathrm{~V})$, was described by Ensenauer et al. [6]. Another novel mutation, c1172G>T, was described by Namik Kaya, et al. [8]. Here we describe a novel homozygous mutation, R332L in the IVD enzyme that presented with a benign phenotype and lack of guidelines for treatment of benign isovaleric acidemia.

\section{CASE REPORT}

A female, full-term infant with normal apgars and normal gestation was thought to have pneumonia 24 hours after birth and was admitted to the intensive care unit. Newborn screening showed an elevated level of C5, $1.34 \mu \mathrm{mol} / \mathrm{L}$ (reference range $<0.30 \mu \mathrm{mol} / \mathrm{L}$ ). Repeated tests showed C5 levels between 2.31 - 9.76 $\mu \mathrm{mol} / \mathrm{L}$ on several occasions. The C5 remained elevated throughout the hospital course and when the baby was well at home. The baby and she did not have the offensive smell typical of IVD, did not develop ketosis, acidosis, or cytopenia. The baby continued to have elevated C5 levels. The stable metabolite of IVA 
in urine, isovalerylglycine, was found to be markedly elevated in this baby, $>150 \mathrm{mmol} / \mathrm{mol}$ creatinine. Other metabolites, $3-\mathrm{OH}$ and $4-\mathrm{OH}$ valerate, were not elevated.

Currently, the baby is seven months old, growing normally, and gaining weight normally. She is developmentally appropriate for age. She has not had any metabolic crises. She is clinically asymptomatic.

\section{IVD Gene Analysis}

Gene analysis was performed by GeneDx, Gaithersburg, MD. Genomic DNA from the patient was PCR amplified for analysis of exons 1-12 and their flanking splice sites on two separate samples to validate the findings.

An unreported mutation $\mathrm{G}>\mathrm{T}$ nucleotide substitution in exon 10 resulted in a missense mutation changing the arginine codon (CGC) with a leucine codon (CTC) at amino acid 332, p.Arg332 L. At the DNA level it is c. $995 \mathrm{G}>\mathrm{T}$. No other sequence changes were discovered in the IVD gene. The patient was found to be homozygous for this mutation.

\section{Diagnosis}

Isovaleric Acidemia, benign phenotype. Genotype A332L/A332L.

\section{DISCUSSION}

The level of C5-acylcarnitine via MS/MS is the primary screening marker for isovaleric academia [9]. Second tier testing to confirm diagnosis relies on the quantification of the levels of isovalerylcarnitine and isovalerylglycine (IVG) in the blood and urine respectively [2] in addition to mutation analysis. Nearly half of IVD mutant alleles detected by newborn screening have been the $932 \mathrm{C} \rightarrow T$ mutation [10]. This mutation is associated with a metabolically mild or intermediate phenotype previously described by Vockley and Ensenauer as C5-acylcarnitine levels between 0.8-6 $\mu \mathrm{mol} / \mathrm{L}$, and IVG levels between 15-195 $\mathrm{mmol} / \mathrm{mol}$ creatinine [3]. More recently however, Enseneaur et al., has proposed a revised C5 concentration range of $0.8-4.8 \mu \mathrm{mol} / \mathrm{L}$ for the identification of metabolically mild or intermediate IVA [11].

For the treatment of IVA, restriction of daily protein as well as dietary supplementation with glycine and carnitine to enhance conjugation and excretion of isovaleric metabolites is recommended (Lin et al., 2007). The baby in this case was given glycine 100 $\mathrm{mg} / \mathrm{kg} / \mathrm{day}$ and carnitine $50 \mathrm{mg} / \mathrm{kg} /$ day. Although treatment may have not been needed, there is uncertainty about the course of the benign forms of IVA. In the study by Dionisi-Vici et al. [5], the clinical course of seven patients identified as having IVA per the newborn screen was more stable than patients diagnosed clinically, with 5 of the 7 never requiring care for metabolic crises. Patients with the $932 \mathrm{C} \rightarrow T$ (A282V) mutation have also remained asymptomatic during a five-year follow up [3].

Ensenauer et al. has proposed defining "metabolically severe" as newborn screen C5 concentrations greater than 8.0-22.1 $\mu \mathrm{mol} / \mathrm{L}$ and using this marker as a basis for treatment decisions [11]; however, there remains concern that metabolic decompensation may occur in the setting of acute illness or physical stress. In 2003, a previously healthy 18-year-old U.S. Air Force recruit was diagnosed with IVA metabolic crisis after developing severe nausea, vomiting, and an anion gap metabolic acidosis during basic training [12]. Given the possibility that others with mild or intermediate phenotypes may experience periods of metabolic crises when under duress, it has been suggested treatment should include low dose carnitine supplementation as needed and careful clinical observation during periods of stress [3].

With expanded newborn screening (NBS), more benign forms of diseases that we recognize in their classical manifestations as severe will be discovered, and such phenotypes will be clarified with long-term follow up. Since the advent of NBS, the incidence of isovaleric academia has been found to be significantly higher in the screened population than in clinically detected cases [5]. This phenomenon has also been seen in other metabolic disorders such as phenylketonuria. Experience with PKU has led to the determination that patients with phenylalanine levels below $360 \mu \mathrm{mol} / \mathrm{L}$ will not require treatment or dietary restrictions [13] despite positive identification with newborn screening. This phenomenon is not unique to IVA and PKU; mild forms of citrullinemia, and very long-chain acyl-CoA dehydrogenase deficiency have also been identified [14]. It is critical that as more and more infants are identified with severe metabolic disorders per the newborn screen, clinicians are made aware that these benign phenotypes exist in order to prevent unnecessary anxiety on the part of the parents, as these patients often show no clinical symptoms. 
Severe forms of PKU, not detected via NBS, may remain undiagnosed until six months of age or older [15]. This is not true, however, for metabolic disorders such as isovaleric academia, maple syrup urine disease and other organic acidemias, which follow a much stormier initial course. It is suggested that patients identified with a benign form of these severe diseases be treated initially with continued follow up during the first years of life. Follow up should include measurement of metabolite levels of isovaleryl-CoA, specifically isovalerylcarnitine and isovalerylglycine. Additionally, special attention should be paid during periods of metabolic crisis as it is unknown if the predisposition for metabolic decomposition exists during periods of stress in patients with benign IVA.

With the discovery of hyperphenylaninemia, a database that correlates $\mathrm{PAH}$ mutations with phenotype has been established, allowing clinicians to better predict the clinical and biochemical phenotype in patients with known genotypes [16]. As with PKU, it is important that a registry of known mutations in the IVD gene be established. It is likely that heterozygotes and compound heterozygotes with benign mutations will follow a benign course as has been with other metabolic disorders and therefore will require little, if any medical intervention. As more and more infants are identified as having IVA per the expanded newborn screen, the ability to reference a patient's genotype in order to determine the biochemical phenotype will better allow clinicians to determine the degree of treatment and follow up required, given that these benign phenotypes do exist.

The C5-acylcarnitine levels of this patient ranged between $2.31-9.76 \mu \mathrm{mol} / \mathrm{L}$, but the baby did not exhibit any clinical signs of severe IVA. It is possible that $\mathrm{C} 5$ levels in individuals with a benign phenotype may fluctuate in the range of what one would consider severe IVA, perhaps in relation to the degree of metabolic stress. Routine monitoring of C5acylcarnitine and urine organic acids should be performed on asymptomatic individuals with the addition of supplemental carnitine and glycine on an as needed basis.

We did not consider this mutation as polymorphic because of the persistent high level of isovaleric metabolites in blood and urine. We have not performed residual activity of IVD. Residual enzyme activity is not routinely done as previous studies have demonstrated a poor correlation between residual IVD activity and disease severity [17]. There is uncertainty about the cases of Ensenauer et al. as long follow up may be needed in order to determine the possibility of metabolic decompensation during a period of catabolic disease.

\section{CONCLUSION}

We describe a novel mutation in the IVD gene, genotype A332L/A332L, that is associated with a benign phenotype.

There is uncertainty about how long patients with mild phenotype of IVA need to be followed since metabolic decompensation during a period of catabolic disease may occur. Recommended therapeutic guidelines at this time for mild or intermediate IVA, defined by Enseneaur et al. as a C5 concentration range of $0.8-4.8 \mu \mathrm{mol} / \mathrm{L}$, include initial treatment with carnitine and glycine, routine monitoring of C5 and urine organic acid levels, and close monitoring with additional support during periods of metabolic stress or illness. Furthermore, we suggest routine genotyping of patients with IVA in order to establish a registry of mutations similar to those in existence for other genetic disorders. This registry will help determine which mutations are associated with a benign biochemical phenotype so that clinicians can formulate better treatment plans for infants identified with IVA via the expanded newborn screen.

There are similar registries that exist for biotinidase deficiency, MCAD, galactosemia, and others.

\section{ABBREVIATION}

\section{IVA = Isovaleric Acidemia}

\section{REFERENCES}

[1] Budd MA, Tanaka K, Holmes LB, Efron ML, Crawofrd JD, Isselbacher KJ. Isovaleric academia: clinical features of a new genetic defect of leucine metabolism. N Engl J Med 1967; 277: 321-327.

\section{http://dx.doi.org/10.1056/NEJM196708172770701}

[2] Ensenauer R, Vockley J, Willard J, et al. A common mutation is associated with a mild, potentially asymptomatic phenotype in patients with isovaleric academia diagnosed by newborn screening. Am J Hum Genet 2004; 75: 1136-1142. http://dx.doi.org/10.1086/426318

[3] Vockley J, Ensenauer R. Isovaleric academia: new aspects of genetic and phenotypic heterogeneity. Am J Med Genet C Semin Med Genet 2006; 142C(2): 95-103.

\section{http://dx.doi.org/10.1002/ajmg.c.30089}

[4] Tanaka K. Isovaleric academia: personal history, clinica survey an study of the molecular basis. Progress in Clinical and Biological Research 1990; 321: 273-290.

[5] Dionisi-Vici C, Deadato F, Roschinger W, Rhead W, WIlcken B. 'Classical' organic acidurias, propionic aciduria, methylmalonic aciduria, and isovaleric aciduria: Long-term 
outcome and effects of expanded newborn screening using tandem mass spectrometry 2006; 29: 383-389.

[6] Wilcken B. The consequences of extended newborn screening programmes: do we know who needs treatment? J Inherit Metab Dis 2008; 31: 173-177. http://dx.doi.org/10.1007/s10545-008-0843-8

[7] Wilcken B. Expanded newborn screening: reducing harm and assessing benefit. J Inherit Metab Dis 2010; 33(Suppl 2): S205-S210.

http://dx.doi.org/10.1007/s10545-010-9106-6

[8] Kaya N, Colak D, Al-Bakheet A, et al. Identification of a novel IVD mutation in a consanguineous family with isovaleric acidemia. Gene 2013; 513: 297-300. http://dx.doi.org/10.1016/i.gene.2012.09.097

[9] Shigematsu Y, Hata I, Tajima G. Useful second-tier tests in expanded newborn screening of isovaleric academia and methylmalonic aciduria. J Inherit Metab Dis 2010; 33(Suppl 2): S283-S288. http://dx.doi.org/10.1007/s10545-010-9111-9

[10] Ensenauer R, He M, Willard JM, Goetzmann ES, Corydon TJ, Vandahl BB, Mohsen AW, Isaya G, Vockley J. Human acyl-CoA dehydrogenase- 9 plays a novel role in the mitochondrial beta-oxidation of unsaturated fatty acids. J Biol Chem 2005; 280: 32309-32316. http://dx.doi.org/10.1074/jbc.M504460200

[11] Ensenauer R, Fingerhut R, Maier E, Polanetz R, Olgemoller B, Roschinger W, Muntau A. Newborn screening for isovaleric academia using tandem mass spectrometry: data from 1.6 million newborns. Clinical Chemistry 2011; 57(4): 623-626.

http://dx.doi.org/10.1373/clinchem.2010.151134

[12] Feinstein J, O'Brien K. Acute metabolic decompensation in an adult with isovaleric academia. Southern Med Journal 2003; 96: 500-503.

http://dx.doi.org/10.1097/01.SMJ.0000051141.03668.1D

[13] Campistol J, Gassio R, Artuch R, Vilaseca MA. Neurocognitive funtion in mild hyperphenylalaninemia. Dev Med Child Neurol 2011; 53: 405-408.

http://dx.doi.org/10.1111/j.1469-8749.2010.03869.x

[14] Levy HL. Newborn screening conditions: What we know, what we do not know, and how we will know it. Genet Med 2010; 12(12): S213-S214. http://dx.doi.org/10.1097/GIM.0b013e3181fe5d77

[15] Matalon R, Michals K. Phenylketonuria: Screening, treatment, and maternal PKU. Clin Biochem 1991; 24: 337342. http://dx.doi.org/10.1016/0009-9120(91)80008-Q

[16] Ponzone A, Spada M, Roasio L, Porta F, Mussa A, Ferraris S. Impact of neonatal protein metabolism and nutrition on screening for phenylketonuria. J Pediatr Gastroenterol Nutr 2008; 46(5): 561-569. http://dx.doi.org/10.1097/MPG.0b013e31815eead6

[17] Vockley J, Parimoo B, Tanaka K. Molecular characterization of four different classes of mutations in the isovaleryl-CoA dehydrogenase gene responsible for isovaleric academia. Am J Hum Genet 1991; 40: 147-157. 